\title{
Preoperative prediction of prosthesis size using cross sectional echocardiography in patients requiring aortic
} valve replacement

\author{
A MACKAY, M BEEN, E RODRIGUES, J MURCHISON, D P DE BONO \\ From the Department of Cardiology, Royal Infirmary, Edinburgh
}

SUMMARY In 43 patients who underwent aortic valve replacement for aortic stenosis with or without regurgitation the accuracy of preoperative left ventricular angiography, parasternal long axis cross sectional echocardiography of left ventricular outflow tract and proximal ascending aorta, and $M$ mode echocardiography of aortic root in predicting aortic root size and thereby prosthesis size was compared. Cross sectional echocardiographic measurements and angiographic measurements of aortic root correlated well with prosthesis size, with over two thirds of the indirect measurements being within $2 \mathrm{~mm}$ of prosthesis diameter. $M$ mode echocardiography did not yield useful predictive information. Non-invasive preoperative evaluation of patients likely to require aortic valve replacement may be usefully extended to include aortic root dimensions measured by cross sectional echocardiography.

Surgical replacement of the aortic valve is the definitive treatment of severe aortic stenosis or regurgitation in adults. It is preferable to perform aortic valve replacement electively, even in the absence of severe symptoms, rather than to await decompensation and the less predictable results of emergency surgery. This requires physicians and surgeons to consider all relevant information relating to the advisability, timing, and technique of the procedure. The invasive investigation of patients with aortic stenosis by cardiac catheterisation causes a small but measurable risk, and there is increasing interest in the possibility that adequate preoperative information might be obtained by non-invasive techniques such as echocardiography. ${ }^{12}$ Echocardiography is useful in the diagnosis of aortic stenosis and regurgitation and, with certain limitations, in assessing their severity. ${ }^{3-5}$ It can give an estimate of left ventricular function and identify associated lesions of other valves. Its inability to detect coronary lesions may not be a major handicap in the absence of angina. ${ }^{2}$

We considered that an additional factor, the size of the aortic root, should also be assessed preoperatively.

Requests for reprints to Dr D P de Bono, Department of Cardiology, Royal Infirmary, Edinburgh EH3 9YW.

Accepted for publication 15 January 1985
Thus in this study we compared angiographic, $\mathbf{M}$ mode echocardiographic, and cross sectional echocardiographic preoperative assessments of aortic root size with the findings at surgery and the size of the valve prosthesis used in $\mathbf{4 3}$ patients undergoing aortic valve replacement.

\section{Patients and methods}

Forty three patients with aortic stenosis with or without aortic regurgitation who underwent elective aortic valve replacement at this hospital were studied. Thirty were men, 13 women. Their ages at the time of operation ranged from 23 to 72 (mean 57.6) years. In addition to aortic valve replacement four patients also underwent coronary artery bypass graft surgery, four mitral valve replacement, two open mitral valvotomy, and one pericardectomy. Of the aortic valves implanted, 17 were Björk-Shiley prostheses (19$31 \mathrm{~mm}$ ), 13 Ionescu-Shiley prostheses $(21-27 \mathrm{~mm})$, seven Hancock prostheses (21-29 mm), and six Carpentier-Edwards prostheses (23-25 mm). In each case the internal diameter of the aortic annulus was measured with a plastic sizer before the prosthesis was sutured in situ. All prostheses were implanted at the level of the aortic annulus.

As part of their preoperative assessment all 


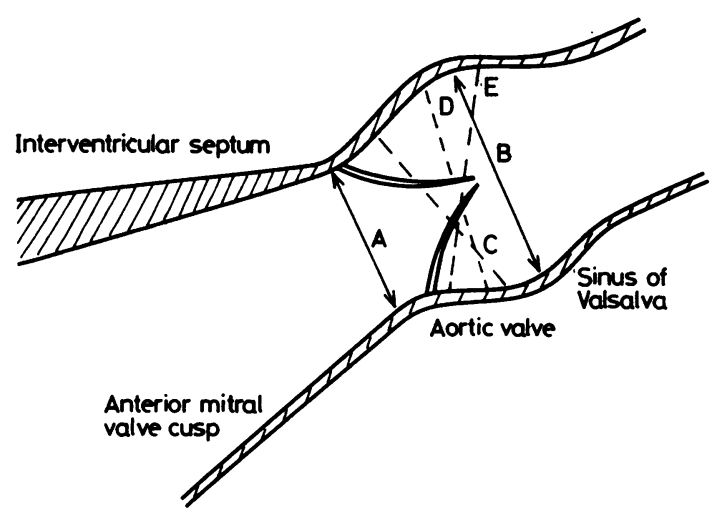

Fig. 1 Diagram showing basis of echocardiogram measurements on parasternal long axis view of left ventricular. outflow tract and proximal ascending aorta. $(A)$ delineates aortic root measurement from luminal surface to luminal surface, and (B) the diameter at the sinus of Valsalva level. $(C),(D)$, and (E) (broken lines) show the possible variations of $M$ mode measurements of aortic root size depending on the siting and angulation of the transducer.

patients underwent $M$ mode and cross sectional echocardiography and cardiac catheterisation. Echocardiography was performed using an Irex System III real time phased array imaging system (Irex Medical Systems, New Jersey, USA) with patients lying supine or in the left semilateral position. A 2.5 $\mathrm{MHz}$ transducer was used to obtain cross sectional parasternal long axis views of the left ventricle, left ventricular outflow tract, aortic valve, and proximal ascending aorta, as well as short axis views of the left ventricle and aortic root. Measurements were made on the long axis recordings. A $2 \cdot 25 \mathrm{MHz}$ transducer was used to obtain $M$ mode recordings of the aortic root. In some patients calcification in the region of the aortic root and valve made cross sectional $(n=4)$ or $M$ mode $(n=14)$ records unsuitable for quantitative analysis. Left ventricular angiography in the $30^{\circ}$ right anterior oblique projection was performed by standard techniques using Urografin 370. Angiograms were recorded in all except eight patients, in whom a severely disorganised valve could not be crossed.

Four observers participated in the study. Each record was measured in triplicate by two observers independently. Measurements were made on cross sectional echocardiograms and left ventricular angiograms according to a predetermined protocol. Aortic root size (A) (Fig. 1) was measured from luminal surface to luminal surface, from the point where interventricular septum became anterior aortic root to the point where anterior mitral valve cusp became posterior aortic root. Further measurements (B) (Fig. 1) were taken parallel to the root measurements at the point of maximum aortic diameter in the region of the
Table Correlation of indirectly measured aortic root dimensions with prosthesis valve size

\begin{tabular}{lll}
\hline Techrique and measurement & $\begin{array}{l}\text { Correlation } \\
\text { coefficient }\end{array}$ & $p$ value \\
\hline Cross sectional echocardiogram: & 0.68 & $<0.001$ \\
$\quad$ Root size & 0.45 & $<0.02$ \\
$\quad \begin{array}{l}\text { Sinus of Valsalva size } \\
\text { mode echocardiogram: }\end{array}$ & 0.22 & $>0.05$ \\
$\quad$ Valve level & 0.62 & $<0.001$ \\
Left ventricular angiogram: & 0.58 & $<0.001$ \\
$\quad$ Root size & Sinus of Valsalva size & 0 \\
\hline
\end{tabular}

sinuses of Valsalva, again from luminal surface to luminal surface. $M$ mode measurements were taken at the aortic valve level from luminal surface to luminal surface at the end of ventricular diastole.

Linear regression analysis was used to compare indirect measurements of aortic size with the dimensions of the prosthetic valves at subsequent surgery.

\section{Results}

There was good correlation (Table) between the size of the aortic annulus, as assessed at surgery and reflected in prosthesis size, and the angiographic or cross sectional echocardiographic estimates of aortic root size (Fig. 2) (coefficients of correlation $0.62,0.68$ respectively). Indirect measurements of root size tended to underestimate prosthesis size, but the differences were not large. Seventy two per cent of cross sectional echocardiogram measurements $(n=39)$ and $70 \%$ of angiographic measurements $(n=35)$ were within $2 \mathrm{~mm}$ of the prosthesis size used at operation. Cross sectional echocardiography also detected two

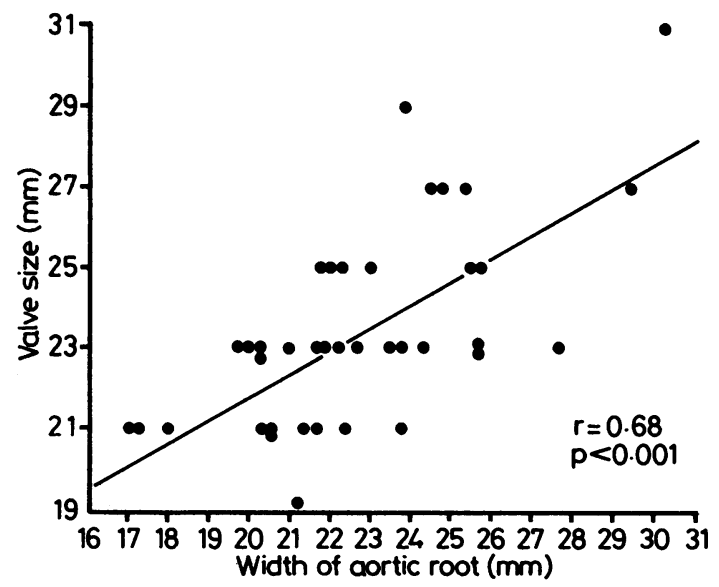

Fig. 2 Correlation of cross sectional echo measurements of aortic root size with prosthesis dimensions at subsequent surgery. $y=0.57 x+10.4 ; n=39 ; r=0.68 ; p<0.001$. 
cases of subvalvar aortic stenosis associated with valvar stenosis.

There was little variation between the observations of pairs of observers reporting both cross sectional echocardiographic (coefficient of correlation 0.94, 0.91 ) and angiographic (coefficient of correlation 0.89 , 0.92 ) data.

M mode assessments were less closely correlated with prosthesis size, only $42 \%$ of $\mathrm{M}$ mode meaasurements lying within $5 \mathrm{~mm}$ of the prosthesis size used.

\section{Discussion}

The optimum choice of aortic valve prosthesis is still controversial. Mechanical prostheses have a longer track record than xenografts or pericardial tissue valves, particularly in young patients, but are haemodynamically less efficient. Use of the smaller size of mechanical prosthesis may be associated with a persisting left ventricular outflow gradient after surgery, so called patient-prosthesis mismatch. ${ }^{6}$ Techniques are available to increase aortic root diameter by inserting a gusset, ${ }^{78}$ but they increase operation time and the risk of haemorrhagic complications. It is also possible to insert a valve in a supra-annular position, when special care is needed not to obstruct the coronary ostia. Preoperative knowledge of the size of the aortic root will be a factor that will help to predict the requirements of individual patients. In turn, knowledge of the type of valve or procedure likely to be needed may influence the timing of surgery. It is also important for the surgeon to know of any subvalvar aortic obstruction since failure to relieve this will negate the effects of valve replacement. Finally the extent of calcification in the aortic valve ring and its possible extension into the crus cordis may be relevant information in predicting the ease and safety of valve replacement.

The present study indicates that real time cross sectional echocardiography and angiography correlate well in their estimates of aortic root size and are equally effective in predicting the size of the prosthesis that may be used but that $M$ mode echocardiography is less effective. It is recognised that assessment of aortic root size at surgery using a sizer is prone to inaccuracy, but, nevertheless, it is a practical procedure related to the size of prosthesis to be implanted. A further potential source of error might be supra-annular positioning of prostheses, but in this study in no instance did the surgeon record implantation of a prosthesis at a site other than that of the aortic annulus. Our echocardiographic findings are in agreement with those of a recent study in which a small number of patients with aortic stenosis was studied, ${ }^{9}$ while the value of invasive investigation by angiography in determining aortic root size has been previously demonstrated. ${ }^{10}$

Cross sectional echocardiography is also effective in providing information about subvalvar stenosis and calcification of the crus cordis, but technical factors related to the shape of the chest and the disposition of the heart may prevent satisfactory recordings being obtained in all patients.

$M$ mode echocardiography has been regarded as a reliable technique for measuring aortic root diameter in normal subjects or those with non-calcific aortic valve disease. ${ }^{11}$ Its lack of efficacy in the present study largely reflects the difficulty in orientating the transducer reliably (Fig. 1) in the presence of multiple strong echoes from calcific material and in deciding the precise boundaries of the aortic root. ${ }^{12}$

In summary, we have found that either angiographic or cross sectional echocardiographic measurements of aortic root size can help to predict the size of prosthesis that can be accommodated, and we suggest that their measurement should be part of the routine assessment of patients for aortic valve replacement. The echocardiographic measurements may be particularly useful when valve replacement is undertaken without prior angiography, a course which has been increasingly supported for patients without angina. ${ }^{2}$

\section{References}

1 St John Sutton MG, St John Sutton M, Oldershaw, P, et al. Valve replacement without preoperative cardiac catheterisation. $N$ Engl f Med 1981; 305: 1233-8.

2 Borow KM, Wynne J, Sloss LJ, Cohn LH, Collins JJ. Non invasive assessment of valvular heart disease: surgery without catheterization. Am Hean f 1983; 106: 443-9.

3 Bennett DH, Evans DW, Raj MVJ. Echocardiographic left ventricular dimensions in pressure and volume overload. Their use in assessing aortic stenosis. Br Heart $\mathcal{F}$ 1975; 37: 971-7.

4 Reichek N, Devereux RB. Reliable estimation of peak left ventricular systolic pressure by $M$-mode echographic-determined end-diastolic relative wall thickness: identification of severe valvular aortic stenosis in adult patients. Am Heart f 1982; 103: 202-9.

5 Motro M, Vered Z, Rath S, Schneeweiss A, Neufeld HN. Correlation between echocardiography and cardiac catheterization in assessing the severity of aortic stenosis. Eur Heart $\mathcal{J}$ 1983; 4: 117-20.

6 Rahimtoola SH. The problem of valve prosthesis-patient mismatch. Circulation 1978; 58: $20-4$.

7 Blank RH, Pupello DF, Bessone LN, Harrison EE, Sbar S. Method of managing the small aortic annulus during valve replacement. Ann Thorac Surg 1976; 22: 356-61.

8 Manouguian S, Seybold-Epting W. Patch enlargement of the aortic valve ring by extending the aortic incision into the anterior mitral leaflet. I Thorac Cardiooasc Sung 1979; 78: 402-12.

9 Cohen JL, Austin SM, Kim CS, Christakos ME, Hussain SM. Two-dimensional echocardiographic preoperative prediction of prosthetic artic valve size. Am Hear f 1984; 107: 108-12.

10 Babb JD, Parr GVS, O'Neill MJ Jr. Predicting aortic valve prosthesis size: a step toward better matching of patient and prosthesis. I Thorac Cardiovase Surg 1981; 81: 450-4.

11 Francis GS, Hagan AD, Oury J, O'Rourke RA. Accuracy of echocardiography for assessing aortic root diameter. $\mathrm{Br}$ Heart $\mathrm{f}$ 1975; 37: 376-8.

12 Reeves WC, Ettinger U, Thomson K, et al. Limitations in the echocardiographic assessment of aortic root dimensions in the presence of aortic valve disease. Radiology 1979;.132: 411-3. 